\title{
Ultra-Violet Treatment for Fermenting Low-Salt Soya Sauce
}

\author{
Sophia Ferng ${ }^{1}$, I-Hui $\mathrm{Wu}^{1}$, Ang-Yen $\mathrm{Li}^{2}$, Cheng-Kuang $\mathrm{Hsu}^{1}$, \\ Robin Yih-Yuan Chiou' ${ }^{1}$, Ching-Hua Ting ${ }^{2, *}$ \\ ${ }^{1}$ Department of Food Science, National Chiayi University, Chiayi, Taiwan \\ ${ }^{2}$ Department of Mechanical and Energy Engineering, National Chiayi University, Chiayi, Taiwan \\ Received 22 April 2019; received in revised form 17 May 2019; accepted 26 August 2019 \\ DOI: https://doi.org/10.46604/peti.2020.4117
}

\begin{abstract}
Low-salt soya sauce has become a market trend due to consumers' demand for a low sodium diet life. In tradition, a low-salt soya sauce (with salt concentration below 14.4\%) is made from a high-salt one (18\% salt concentration) through diluting or reducing the sodium content. The post-processing deteriorates the quality of the soya sauce produce as some specific, beneficial chemical components are inevitably removed. In the production of a native-born low-salt soya sauce, a key problem encountered is possible microbial contamination that easily develops in a low salt environment. In this study, we evaluated the effect of ultra-violet (UVC $254 \mathrm{~nm}$ ) irradiation on soya mash of $12 \%$ salt concentration fermented at $35^{\circ} \mathrm{C}$. The ultra-violet treatment could effectively prevent the soya mash from microbial contamination.
\end{abstract}

Keywords: soya sauce, law salt, ultra-violet treatment, native-born

\section{Introduction}

Soya sauce was invented by the Chinese about 3500 years ago. It has become a popular condiment on a table or seasoning in cooking in the oriental countries, as it not only provides a delicious flavor to the food but also promotes digestion to the body [1]. It can be made from a mixture of soya beans and wheat using a well-established two-step fermentation process [2], i.e. koji and moromi fermentation. In moromi fermentation, the mash can be easily spoiled because of microbial contamination by unexpected microorganisms that are carried by the materials or mostly come from the atmosphere [3]. To avoid microbial contamination during fermentation, the mash fermentation is normally conducted in $18-20 \%$ brine. The resulting broth contains a very high concentration of salt. This leads to a soya sauce produce rich of sodium that may incur cardiovascular and kidney diseases to the human body after serving. For health, soya sauce with a salt content less than $130 \mathrm{~g} / \mathrm{l}$ is recommended [4].

The regular industrial procedure of making low-salt soya sauce is to make a high-salt (18\%) broth and then dilute the broth or reduce the sodium content with certain physical or chemical operations [4]. Both approaches will inevitably deteriorate the quality of the low-salt soya sauce produce as the flavor chemicals and nutrient components are inevitably removed in post-processing.

In tradition, a moromi pot is placed outdoors for fermentation that may take four months to one year [5-6], depending on the climate. The moromi placed outdoors is vulnerable to microbial contamination. Outdoor fermentation is unsuitable for the production of low-salt soya sauce as unexpected microorganisms can grow easily in a low-salt environment. Also, this lengthy fermentation time occupies a big portion of the cost in soya sauce production. The industry has devoted efforts in shortening

* Corresponding author. E-mail address: cting@mail.ncyu.edu.tw

Tel.: +886-5-2717642; Fax: +886-5-2717561 
the time of fermentation. Chemical soya sauce is a typical example in which, the amino acid, an important component of soya sauce is hydrolyzed from soya bean using hydrochloric acid. Amino acid is then conditioned with flavoring compounds and browned with chemicals to produce soya sauce [7].

Humidity and temperature are two climatic factors that dominate the enzymatic activation and microbial metabolism in moromi fermentation. Moromi is sunk in brine, an uncontrollable, high humid environment. The temperature is a parameter sensible to the ambiance which varies hour by hour, day by day, and season by season. The enzymatic activation and microbial metabolism at different phases of fermentation could progress efficiently and nicely under a stable, adequate fermentation temperature profile [8]. The fermentation of moromi could be shortened to three to four months with less or no unexpected microbial contamination under a temperature-controlled environment [9-10]. A temperature-controlled environment has demonstrated its superiority in preventing hazardous microbial contamination [10]. This is because the enzymatic conversion of proteins may act rigorously in the first few days under an adequate fermentation temperature [11].

Most microbes do not survive in a high-salt environment. However, the surface of moromi can be easily contaminated by ambient bacteria as the surface is not sunk in brine and is exposed to the air [3]. To prevent contamination by the microbes from the air, in this study, ultraviolet irradiation at the wavelength $254 \mathrm{~nm}$ (i.e. UVC) was used to eradicate possible microorganisms on the moromi surface. This is beneficial as ultra-violet light does not penetrate into the surface of moromi other than clear water [12]. To evaluate the efficacy of UVC treatment on the production of low-salt soya sauce, moromi of $18 \%$ and $12 \%$ salt concentrations were used as a control group while another $12 \%$ moromi treated with UVC irradiation as the experimental group. The counts of yeast, total viable bacteria, and Bacillus spp. bacteria were examined every two weeks during three months of fermentation to justify the performance of UVC treatment on production of low-salt soya sauce.

\section{Materials and Methods}

\subsection{Raw materials and chemicals}

Fresh soya bean and wheat once bought were immediately stored in a refrigerator at $4^{\circ} \mathrm{C}$ prior to using. The soya bean (Glycine max Shi-Shi) was cultivated by National Chiayi University, Taiwan and the wheat was imported from Canada. The koji mold was Aspergillus oryzae (Chuan Feng Microbe, Taiwan). All chemicals used were of analytical grade.

\subsection{Koji fermentation}

Raw soya bean was first washed and soaked in $4^{\circ} \mathrm{C}$ water for 24 hours and then steamed at $121^{\circ} \mathrm{C}$ for 20 minutes. Wheat was roasted at $140^{\circ} \mathrm{C}$ for 30 minutes and then cracked into four to five pieces per kernel that may accompany with small particles (i.e. wheat flour). Roasted wheat and steamed soya bean were mixed at a ratio of 1:2 (w/w). The mixture was then inoculated with $0.1 \%$ (w/w) of Aspergillus oryzae (ca. 108 spores $\mathrm{g} / \mathrm{l}$ ), and subsequently dispersed onto perforated stainless steel trays $(68 \times 44 \times 3 \mathrm{~cm})$. Each tray was loaded with the mixture to around $3 \mathrm{~cm}$ in thickness and incubated at $30 \pm 3^{\circ} \mathrm{C}$. Koji was harvested when the culture turns into greenish-yellow in color. This process may take 60-72 hours. The koji produce was stored at $4^{\circ} \mathrm{C}$ before subsequent moromi fermentation.

\subsection{Moromi fermentation}

Fig. 1 illustrates the setup of the temperature-controlled fermentation system [10]. Both experimental and control groups used 20-1 pottery tanks for moromi fermentation. Moromi tanks were bathed in a stainless tank (diameter $84 \mathrm{~cm}$ and height 65 $\mathrm{cm}$ ) full of circulating water for temperature control. Temperatures inside and outside of the pottery tank were measured for on-line fermentation temperature control and off-line analysis. Our previous experimentation showed no temperature gradient in the mash at steady state. Hence the mash had only its central temperature being measured. The circulating water was heated to $45^{\circ} \mathrm{C}$ with a heat pump. The fermentation temperature was instructed to maintain at $35^{\circ} \mathrm{C}$, a compromised temperature 


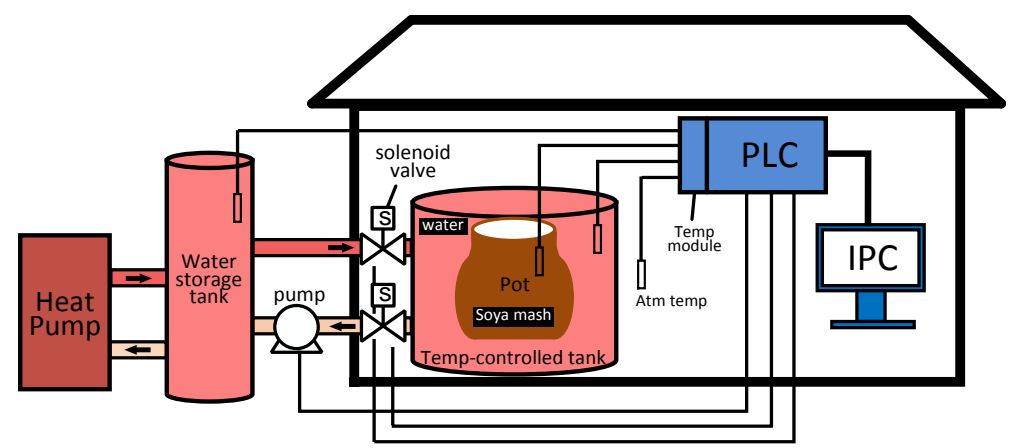

Fig. 1 Setup of the moromi fermentation system [10]

suitable for yeast, lactic acid bacteria, and Aspergillus oryzae, but not for hazardous bacteria or film yeast (Pichia spp.) [9, 13]. A Programmable Logic Controller (PLC) performs controls and an Industrial Personal Computer (IPC) executes system monitoring and data acquisition.

Fermented koji was put in the pottery tanks and then mixed with $18 \%$ or $12 \%$ brine at a weight ratio of $1: 2$. The tanks were covered in compliance with industrial practice except allowing the insertion of thermocouples for temperature measuring. The temperature-controlled system was instructed to maintain the central temperature of the mash at $35 \pm 1^{\circ} \mathrm{C}$. During the first week of fermentation, the mash was stirred for three minutes once every day and then once a week for the remaining period of fermentation. Both groups had a microbial examination every two weeks till the fermentation completed three months after [10].

Fig. 2 illustrates the setup of moromi fermentation under UVC treatment. Moromi in 12\% brine was placed in a 20-1 pottery tank and the tank is covered with a stainless lid, on that, a UVC lamp (16W FAM/10X25BOX, Philips, Holland) and a timer controller were mounted. The timer controls the timing and the duration of UVC irradiation. UVC dosage was therefore properly administered. The distance between the surface of the soya mash and the UVC lamp was well adjusted to allow the moromi surface with uniform UVC irradiation.

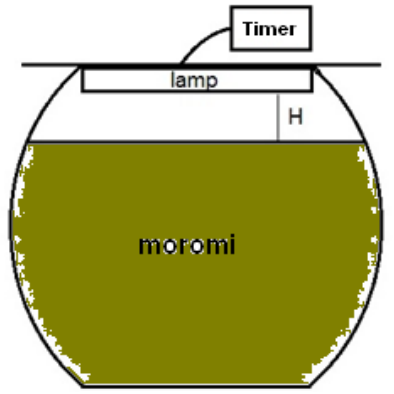

(a) UVC setup

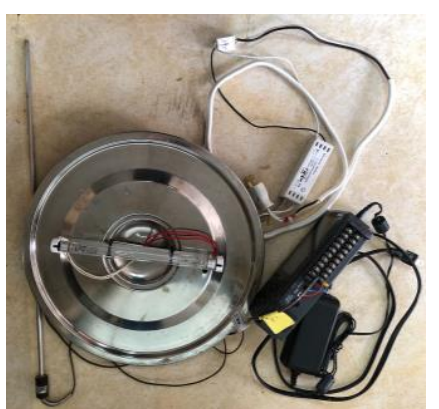

(b) tank lid with a UVC lamp and a temperature sensor mounted

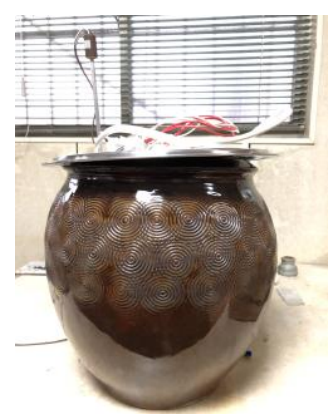

(c) complete setup

Fig. 2 Setup of soya mash fermentation under UVC treatment

\subsection{Microbial analysis}

The unique flavor of moromi is attributed to lactic acid bacteria in the first stage of moromi fermentation, resulting in decreased $\mathrm{pH}$ in moromi to 6 or below, at which yeast starts to propagate [14]. Unexpected extra bacteria, mainly Bacillus spp., may intrude from the ambience in moromi fermentation, form molds on the mash surface, and lead therefore to a stinking broth. Thus, counting the growth facts of the total viable bacteria, the yeast, and the Bacillus spp. bacteria unveils the progress of microbial metabolism and the efficacy of the UVC treatment in fermentation [15]. Microbial analysis was operated every two weeks.

The 10 -fold-concentrated cells were serially diluted $\left(10^{0}\right.$ to $\left.10^{-6}\right)$ in a $0.85 \%$ sterile saline solution. To count the total viable bacteria, $100 \mu \mathrm{l}$ of the properly diluted sample was spread on plate count agar (PCA), incubated at $30^{\circ} \mathrm{C}$ for 48 hours, 
and then counted. For yeast counting, $100 \mu \mathrm{l}$ of the properly diluted sample was spread on YMA (yeast mold agar), incubated at $30^{\circ} \mathrm{C}$ for 48 hours, and then counted. To count the Bacillus spp. bacteria, $100 \mu \mathrm{l}$ of the properly diluted sample were spread on MYP (mannitol egg yolk polymyxin agar), incubated at $30^{\circ} \mathrm{C}$ for $24-48$ hours, and then counted. The colonies formed (25-250) were calculated as log CFU per milliliter of soya sauce [16].

\section{Results}

\subsection{Visual inspection of fermented moromi}

Fig. 3 shows visual inspection of different mature moromi with and without UVC treatment. The moromi with $18 \%$ brine, an ordinary industrial formula, had the darkest color and no mold development on the surface and had the stickiest broth among all groups. The $12 \%$ moromi without UVC treatment had unwanted, hazardous, odorous, grey-greenish molds developed on the surface. Moromi produces with such quality is considered failed. The 12\% moromi with UVC treatment did not have mold development on the surface. The color was less dark and the broth was less sticky than the $18 \%$ one. Mature moromi with such a quality can be processed for further soya sauce production.

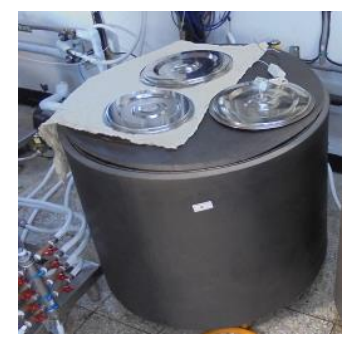

(a) the temperature-controlled tank

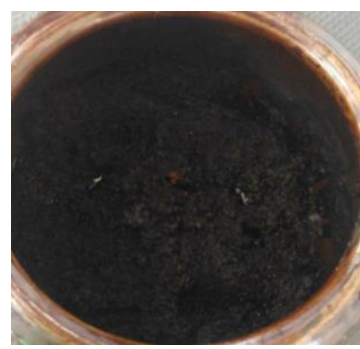

(b) $18 \%$ brine

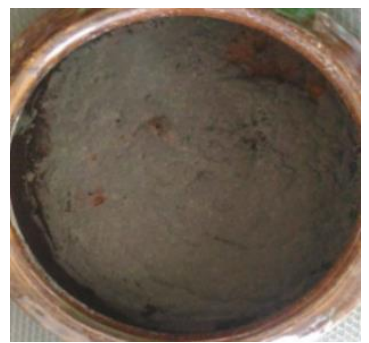

(c) $12 \%$ brine

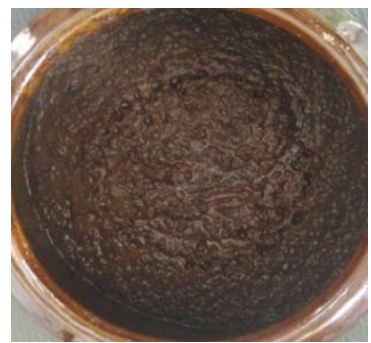

(d) $12 \%$ brine with UVC

Fig. 3 Visual inspection of different mature moromi

\subsection{Optimum UVC dosage}

There is no existing guideline for a proper UVC dosage on the application of soya sauce fermentation. Hence several treatment schemes were evaluated to arrive at optimum dosage. The moromi was exposed to UVC irradiation for one hour every 24 hours. This scheme resulted in a browning reaction on the moromi surface but no mold development was observed on the surface. The browning reaction was unwanted as the mash was "burned" by the UVC. The treatment was adjusted to 30 min irradiation every 12 hours. There was a bit mold development on the moromi surface, but no more browning reaction observed. The dosage was further adjusted to 30 minutes irradiation every six hours. This scheme was acceptable as neither surface contamination nor browning reaction was observed.

\subsection{Microbial Analysis}

Fig. (4)-(6) show the changes in yeast, total viable bacteria, and Bacillus spp. bacteria counts in the course of moromi fermentation. The bacteria were counted every two weeks and broth samples were taken on the surface (suffixed with "S") and in the center of the moromi (suffixed with "C").

The yeast counts (Fig. 4) and the total viable bacteria counts (Fig. 5) decreased slightly at the beginning of fermentation and then saturated. This trend was attributed to the inhibitory effects of salt on microbial growth and the low $\mathrm{pH}$ induced by lactic acid bacteria [17]. The yeast counts did not have a significant difference between the surface and the center positions. Besides, there is no significant difference among all fermentation formula. In general, there were no significant differences in yeast, total viable bacteria, and Bacillus supp. bacteria counts between sampling places and among fermentation schemes. The UVC treatment did not influence the development of known microorganisms in moromi fermentation.

As shown in Fig. 5, each fermentation schemes and sampling places did not have significant differences in the total viable bacteria counts. The surface of the $12 \%$ moromi was contaminated with hazardous bacteria and this lead therefor to visible 
mold development and odorous smell. However, the contamination did not affect the total viable bacteria counts. This might be due to that the salt in the $12 \%$ formula, although much less than the $18 \%$ one, can effectively prevent the contaminating bacteria from further penetrating into the moromi. UVC irradiation does not penetrate the non-transparent object [12]. Hence the viable bacteria in the mash were not affected by the UVC.

Fig. 6 shows the developments of Bacillus spp. bacteria. The Bacillus spp. counts in all schemes increased slightly at the beginning of fermentation and then saturated. There were no significant differences among each moromi schemes. Bacillus spp. bacteria is probiotic, especially beneficial to the digestive system [18]. However, too much Bacillus spp. bacteria in moromi produce disgusting flavor, reduces the utilization of nitrogen, and deteriorates the unique aroma of soya sauce produce.

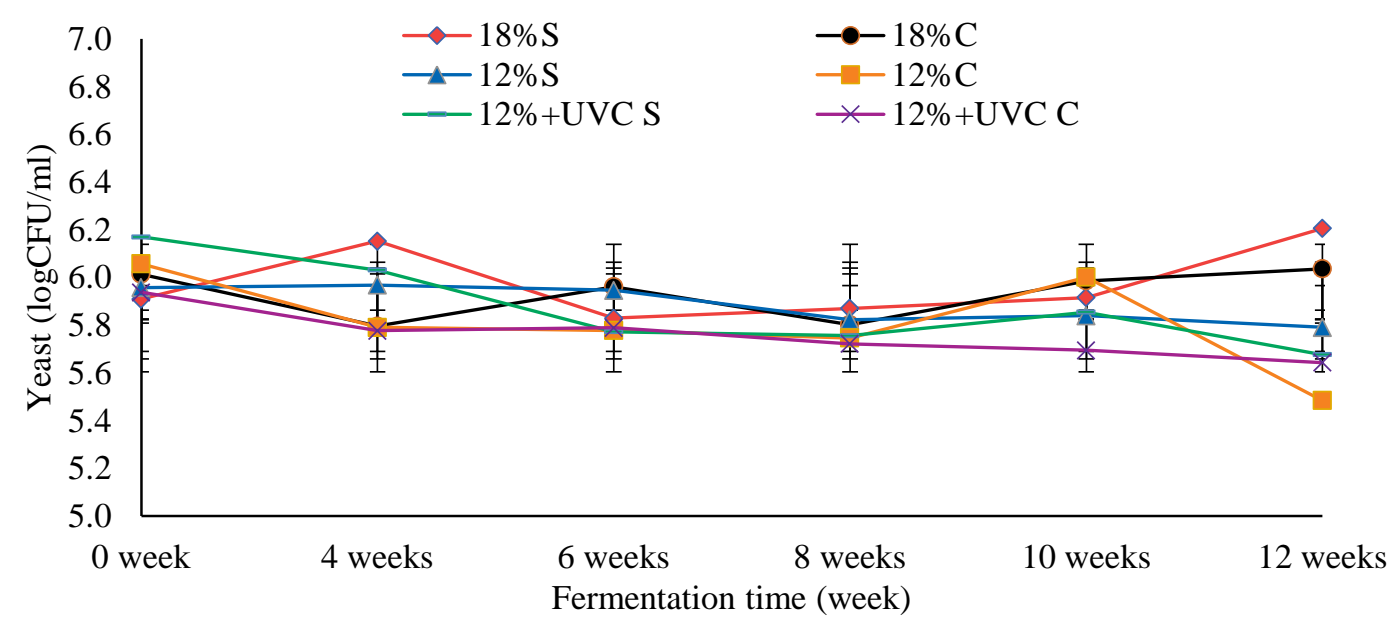

Fig. 4 Development of yeast counts in moromi fermentation

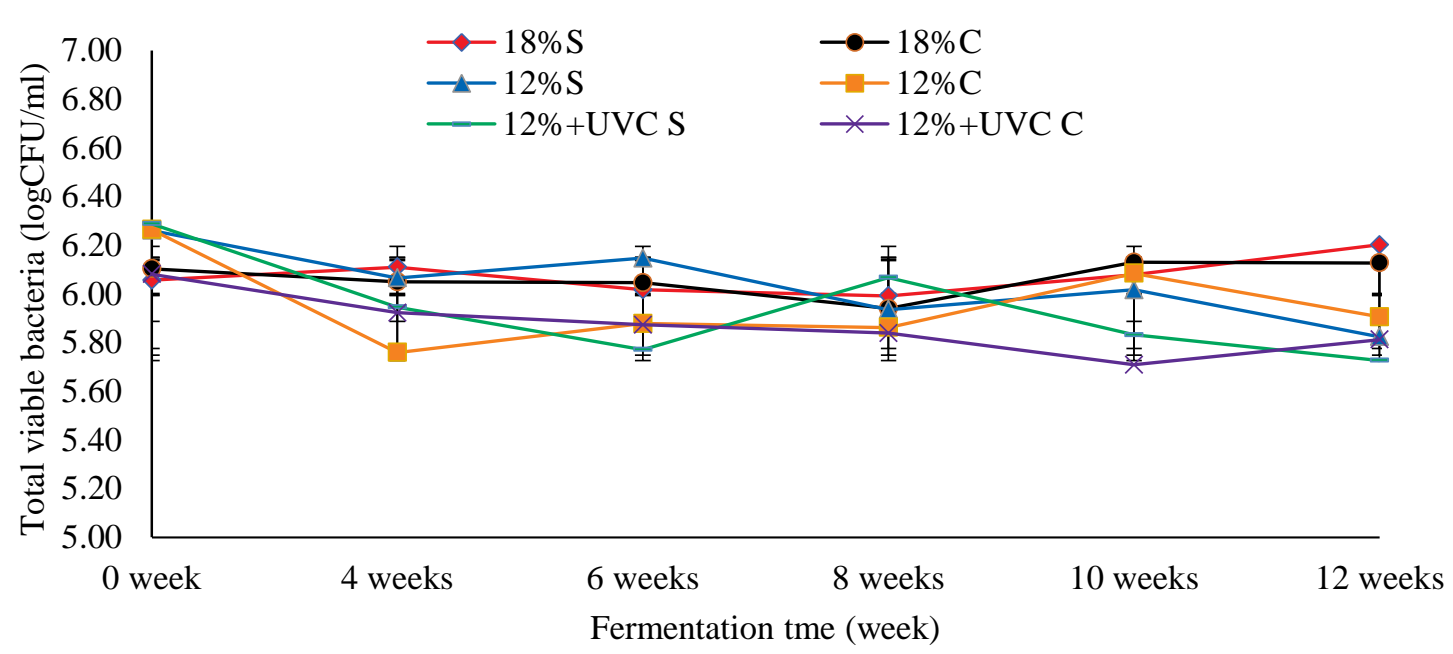

Fig. 5 Development of total viable bacteria counts in moromi fermentation

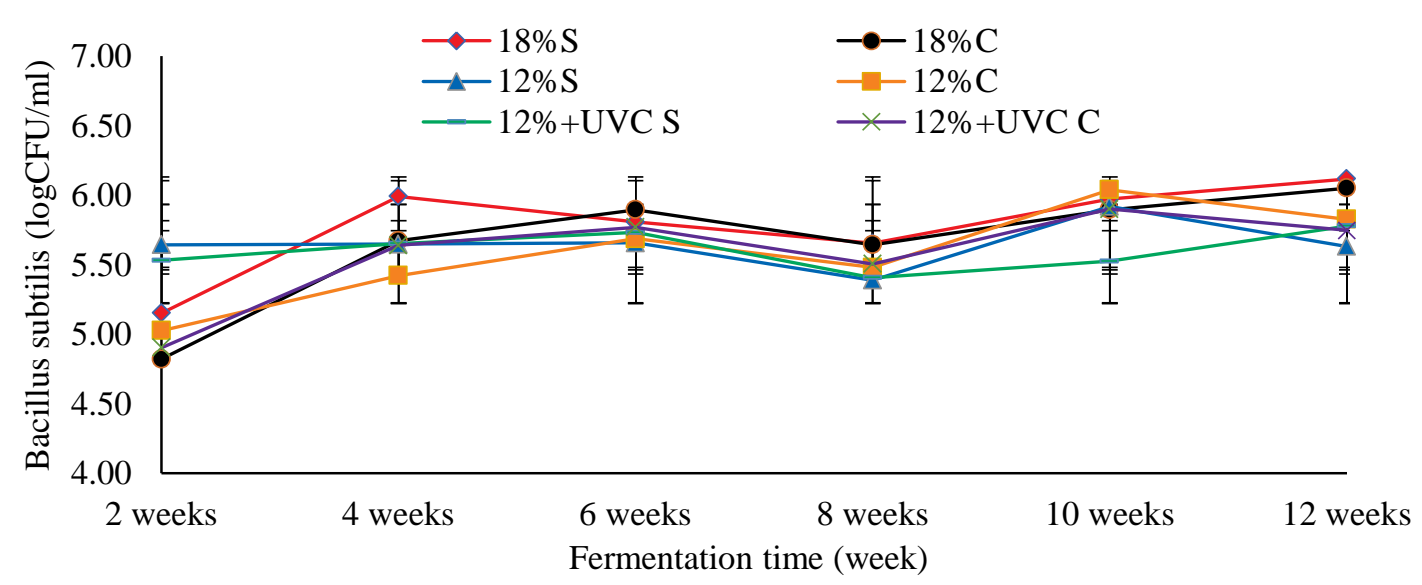

Fig. 6 Development of Bacillus spp. counts in moromi fermentation 


\section{Conclusions}

The purpose of using $18 \%$ bine in moromi fermentation is to inhibit possible hazardous bacteria development as most unwanted bacteria are intolerable to a salt-rich solution. Reducing the salt concentration from $18 \%$ to $12 \%$ incurs the risk of bacterial contamination. The $12 \%$ moromi had serious contamination on the surface, which was unusable in the production of soya sauce. When another $12 \%$ moromi was treated with UVC irradiation, microbial contamination was effectively diminished. Microbial analysis showed that moromi fermentation with UVC treatment did not have a side effect on microbial growth. The optimized UVC dosage (30 minutes irradiation every six hours) could effectively inhibit bacteria development on the surface of moromi and did not induce browning reaction. This dosage can be used for a future application for production of low-salt soya sauce with UVC treatment.

\section{Conflicts of Interest}

The authors declare no conflict of interest.

\section{Acknowledgment}

This work was sponsored by the Ministry of Science and Technology of the ROC (Taiwan) Government under the grant MOST 105-2221-E-415-030.

\section{References}

[1] D. Fukushima, "Soy proteins for foods centering around soya sauce and tofu," Journal of the American Oil Chemists Society, vol. 58, no. 3, pp. 346-354, March 1981.

[2] T. Yokotsuka, "Soya sauce biochemistry,” Advances in Food Research, vol. 30, pp. 195-329, 1986.

[3] W. H. Huang, "Influences of a temperature controlled system powered by solar energy and an UV treatment on the quality of soy sauce," Master Dissertation, Department of Food Science, National Chiayi University, Chiayi, Taiwan, 2016.

[4] J. Luo, L. Ding, X. Chen, and Y. Wan, "Desalination of soy sauce by nanofiltration," Separation and Purification Technology, vol. 66, no. 3, 429-437, May 2009.

[5] J. S. Lee, S. J. Rho, Y. W. Kim, K. W. Lee, and H. G. Lee, "Evaluation of biological activities of the short-term fermented soybean extract," Food Science and Biotechnology, vol. 22, no. 4, pp. 973-978, August 2013.

[6] Y. Zhu and J. Tramper, "Koji-where east meets west in fermentation,” Biotechnology Advances, vol. 31, no. 8, pp. 1448-1457, December 2013.

[7] R. Schueller, The soya sauce handbook: a reference manual for the food manufacturer, Kikkoman Corporation, Japan, 1996.

[8] N. W. Su, M. L. Wang, K. F. Kwok, and M. H. Lee, "Effects of temperature and sodium chloride concentration on the activities of proteases and amylases in soya sauce koji," Journal of Agricultural and Food Chemistry, vol. 53, no. 5, pp. 1521-1525, April 2005.

[9] F. M. Yong and B. J. B. Wood, "Microbiology and biochemistry of soya sauce fermentation," Advances in Applied Microbiology, vol. 17, pp. 157-194, 1974.

[10] S. Ferng, W. H. Huang, C. P. Wu, Y. T. Lu, C. K. Hsu, R. Y. Y. Chiou, and C. H. Ting, “A controlled fermentation environment for producing quality soya sauce," Advances in Technology Innovation, vol. 4, no. 1, pp. 11-20, January 2019.

[11] N. X. Hoang, S. Ferng, C. H. Ting, Y. C. Lu, Y. F. Yeh, Y. R. Lai, and C. K. Hsu, "Effect of initial 5 days fermentation under low salt condition on the quality of soy sauce," LWT-Food Science and Technology, vol. 92, pp. 234-241, June 2018.

[12] J. A. Guerrero-Beltrán and G. V. Barbosa-Cánovas, "Review: advantages and limitations on processing foods by UV light," Food Science and Technology International, vol. 10, pp. 137-147, June 2004.

[13] N. X. Hoang, S. Ferng, C. H. Ting, W. H. Huang, R. Y. Y. Chiou, and C. K. Hsu, "Optimizing the initial moromi fermentation conditions to improve the quality of soya sauce," LWT-Food Science and Technology, vol. 74, pp. 242-250, December 2016.

[14] R. Y. Cui, J. Zheng, J. C. D. Wu, and R. Q. Zhou, "Effect of different halophilic microbial fermentation patterns on the volatile compound profiles and sensory properties of sauce moromi," European Food Research and Technology, vol. 239, no. 2, pp. 321-331, August 2014. 
[15] X. L. Gao, C. Cui, H. F. Zhao, M. M. Zhao, L. Yang, and J. Y. Ren, "Changes in volatile aroma compounds of traditional Chinese-type soy sauce during moromi fermentation and heat treatment, “Food Science and Biotechnology, vol. 19, no. 4, pp. 889-898, August 2010.

[16] M. H. Kang, J. S. Kim, S. J. Park, and T. Shibamoto, “A study on biological and chemical changes in fermented soybeans (meju), grown under organic vs non-organic conditions, during storage,” Journal of Agriculture and Biological Sciences, vol. 3, no. 2, pp. 278-286, March 2012.

[17] Y. Yan, Y. Qian, F. Ji, J. Chen, and B. Han, "Microbial composition during chinese soya sauce koji-making based on culture dependent and independent methods," Food Microbiology, vol. 34, no. 1, pp. 189-195, May 2013.

[18] S. Lee, J. Lee, Y. I. Jin, J. C. Jeong, Y. H. Chang, Y. Lee, Y. Jeong, and M. Kim, "Probiotic characteristics of bacillus strains isolated from korean traditional soy sauce," LWT-Food Science and Technology, vol. 79, pp. 518-524, June 2017.

Copyright $\odot$ by the authors. Licensee TAETI, Taiwan. This article is an open access article distributed under the terms and conditions of the Creative Commons Attribution (CC BY-NC) license (https://creativecommons.org/licenses/by-nc/4.0/). 